\title{
Protective effects of Fructus sophorae extract on collagen-induced arthritis in BALB/c mice
}

\author{
HYOUNG-MIN HAN $^{1}$, SU-HYUN HONG ${ }^{2}$, HEUNG-SIK PARK ${ }^{3}$, JAE-CHUL JUNG ${ }^{3}$, \\ JONG-SIK KIM ${ }^{4}$, YONG-TAE LEE ${ }^{1}$, EUN-WOO LEE ${ }^{5,6}$, YUNG-HYUN CHOI ${ }^{2,5}$, \\ BYUNG-WOO KIM ${ }^{5,6}$, CHEOL-MIN KIM ${ }^{7,8}$ and KYUNG-HWA KANG ${ }^{1}$
}

\begin{abstract}
Departments of ${ }^{1}$ Physiology and ${ }^{2}$ Biochemistry, College of Korean Medicine, Dongeui University, Busan 614-851; ${ }^{3}$ NOVAREX Co., Ltd. Life Science R\&D Institute, Ochang, Chungcheongbuk 363-885; ${ }^{4}$ Department of Anatomy, College of Medicine, Kosin University, Busan 602-703; ${ }^{5}$ Anti-Aging Research Center and Blue-Bio Industry RIC; Departments of ${ }^{6}$ Life Science and Biotechnology, Dongeui University, Busan 614-714;

${ }^{7}$ Research Center for Anti-Aging Technology Development, Busan 609-735; ${ }^{8}$ Department of Biochemistry, College of Medicine, Pusan National University, Yangsan, Gyeongsangnam 626-870, Republic of Korea
\end{abstract}

Received April 19, 2015; Accepted May 1, 2016

DOI: $10.3892 / \mathrm{etm} .2016 .3929$

\begin{abstract}
Styphnolobium japonicum (L.) is utilized in Korean medicine for the treatment of various inflammatory diseases. The aim of the present study was to explore the effects of Fructus sophorae extract (FSE) isolated from the dried ripe fruit of $S$. japonicum (L.) on the development of type II collagen-induced arthritis (CIA) in BALB/c mice. The CIA mice were orally administered FSE or saline daily for 2 weeks. The incidence and severity of disease and the inflammatory response in the serum and the joint tissues were assessed. Macroscopic and histological investigation indicated that FSE protected against CIA development. FSE was associated with a significant reduction in the levels of total immunoglobulin G2a and proinflammatory cytokines and mediators in the serum. In addition, FSE suppressed the gene expression levels of proinflammatory cytokines and mediators, the mediator of osteoclastic bone remodeling, the receptor activator of nuclear factor $\kappa$-B ligand and matrix metalloproteinases in the joint tissues. The present results suggest that FSE may protect against inflammation and bone damage, and would be a valuable candidate for further investigation as a novel anti-arthritic agent.
\end{abstract}

\section{Introduction}

Fructus sophorae is the dried ripe fruit of Styphnolobium japonicum (L.). It has traditionally been used

Correspondence to: Dr Kyung-Hwa Kang, Department of Physiology, College of Korean Medicine, Dongeui University, 52-57 Yangjeongro, Busan 614-851, Republic of Korea

E-mail: ghkang@deu.ac.kr

Key words: Fructus sophorae extract, Styphnolobium japonicum L., type II collagen-induced arthritis for its anti-inflammatory properties, cooling blood and stopping bleeding (using blood cooling hemostatics) in Korean and Chinese medicine (1). Several studies have demonstrated that Fructus sophorae has a preventative effect against osteoclastogenesis and bone loss $(2,3)$ and an inhibitory effect on the production of inflammatory cytokines in osteoblast-like cells (4). However, few attempts have been made to observe the effects of Fructus sophorae on rheumatoid arthritis.

The incidence of rheumatoid arthritis (RA) is $\sim 1 \%$ of the current adult population, and the incidence is increasing in industrialized countries, especially in women $>65$ years of age (5).

RA is characterized by symptoms such as persistent synovitis and systemic inflammation, and consequently leads to the destruction of cartilage and bone. RA is a an autoimmune disease, yet the pathogenic causes of RA have yet to be elucidated.

An important factor that accelerates the pathogenesis of RA is the proinflammatory mediators that are overproduced by infiltrating inflammatory cells in inflamed joints. Within the inflamed RA joint, tumour necrosis factor (TNF)- $\alpha$ is one of the predominant proinflammatory cytokines, which provokes the release of several other proinflammatory cytokines, chemokines, prostaglandin E (PGE)2 and nitric oxide (NO), and has diverse pathologic effects relevant to RA (6-10).

A major factor that promotes bone loss in RA is osteoclastogenesis by the binding of the receptor activator of nuclear factor- $\kappa \mathrm{B}$ ligand (RANKL) to its receptor RANK on osteoclast precursor cells. RANKL serves an important role in osteoclast differentiation and induces the activation of matrix metalloproteinases (MMPs), which lead to the degradation of the extracellular matrix, loss of cartilage and formation of osteoclastic bone resorption pits within the joint (11-13).

Collagen-induced arthritis (CIA) is the most widely investigated autoimmune arthritis model, and has provided a valuable experimental model for assessing the pathogenic mechanisms of human RA (14). 
In the present study using the CIA mouse model, the anti-inflammatory properties and protective effects against bone and cartilage damage of Fructus sophorae were investigated.

\section{Materials and methods}

Animals and Fructus sophorae extract (FSE) preparation. Male BALB/c mice (age, 6 weeks; weight, 20-22 g) were purchased from SAMTAKO Bio Korea (Osan, Korea). Mice were housed in a temperature-controlled room (at $23 \pm 1^{\circ} \mathrm{C}$ ) with a $12: 12-\mathrm{h}$ dark:light cycle, in polystyrene cages at Dongeui University and provided with standard rodent chow and water ad libitum. Mice were cared for according to the Guide for the Care and Use of Laboratory Animals (National Academy Press, Washington, DC, USA). The experimental protocol was approved by the Institutional Animal Research Committee of Dongeui University on Animal Care and Use (approval no. DEU-R2014008), and all efforts were made to minimize animal suffering and reduce the number of animals used for the experiments. FSE containing $20 \%$ isoflavone was provided by NOVAREX (Ochang, Gyeongsangbuk-do, Korea; www.novarex.co.kr). The extract was isolated from the dried ripe fruits of $S$. japonicum (L.) with $60 \%$ ethanol and was stored at $-20^{\circ} \mathrm{C}$ until required for experiments. Briefly, $10 \mathrm{~g}$ of Fructus sophorae (Jesung Pharmaceutical Co., Kyungdong Market, Korea) was crushed into 30-mesh size by using a dry pulverizer. Distilled water was added to the crushed Fructus sophorae to dilute it 10X, and the solution was heated at $121^{\circ} \mathrm{C}$ for $2 \mathrm{~h}$. Subsequently, the solution was cooled to $50^{\circ} \mathrm{C}$, followed by filtering with a 200-mesh filter cloth to remove precipitate and obtain the filtrate. Amylase (Pectinase 100 1, Novo Nordisk A/S, Copenhagen, Denmark) was added to the filtrate at a concentration of $0.5 \%(\mathrm{v} / \mathrm{v})$, and an enzyme reaction was performed at $50^{\circ} \mathrm{C}$ for $16 \mathrm{~h}$. The reaction solution was centrifuged to recover precipitate $350 \mathrm{x} g$ for $10 \mathrm{~min}$ at room temperature, and $60 \%$ ethanol was added to the recovered precipitate. The ethanol mixture was mixed for 30 min until the precipitate was completely dispersed. After mixing, the solution was left for $1 \mathrm{~h}$, then centrifuged to remove precipitate and recover supernatant. The supernatant was concentrated to the volume of 1:10 using a concentrator, and the concentrate was powdered using a spray dryer to obtain $0.3 \mathrm{~g}$ of Fructus sophorae extract powder (yield to crushed material of Fructus sophorae: 3\%).The voucher specimens (accession no. DEU-5) have been deposited at a publicly available Natural Resource Bank of Dongeui University College of Koreanl Medicine.

Induction of CIA and FSE treatment. Bovine type II collagen (CII) (Chondrex, Inc, Redmond, WA, USA) was dissolved overnight at $4^{\circ} \mathrm{C}$ in $0.05 \mathrm{M}$ acetic acid at $4 \mathrm{mg} / \mathrm{ml}$, then the solution was emulsified with an equal volume of complete Freund's adjuvant (CFA; Sigma-Aldrich, St. Louis, MO, USA) in an ice-cold water bath. Arthritis was induced using an intradermal injection of $0.05 \mathrm{ml}$ of the cold emulsion into the base of the tail. Two weeks later, the mice were administered a booster immunization with CII emulsified in CFA in the same manner.

Following the onset of CIA, mice were randomized into four groups ( $\mathrm{n}=5 /$ group) as follows: i) Non-immunized (normal control), ii) CII-immunized (CIA control), and CII-immunized FSE treated groups administered iii) 70 or iv) $350 \mathrm{mg} / \mathrm{kg}$ FSE.
Table I. Clinical scores of CIA.

Grade Clinical features

$$
\begin{aligned}
& \text { Paw } \\
& 0 \quad \text { No swelling and redness } \\
& 1 \text { Slight swelling and redness in small joints }{ }^{\mathrm{a}} \text { or large } \\
& \text { joints }^{\text {b }} \\
& 2 \text { Moderate swelling and redness in } \geq 1 \text { joints } \\
& 3 \text { Severe swelling and redness in large joints and moderate } \\
& \text { swelling and redness in small joints } \\
& 4 \text { Most severe swelling and redness in large joints and } \\
& \text { severe swelling and redness in small joints }
\end{aligned}
$$

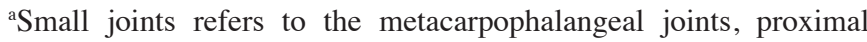
interphalangeal joints, second through fifth metatarsophalangeal joints and thumb interphalangeal joints. ' ${ }^{b}$ arge joints refers to wrists, knees and ankles. The clinical severity of arthritis in each paw and tail was scored by 3 observers, and yielded a maximum possible score of 19 per animal. CIA, type II collagen (CII)-induced arthritis.

FSE dissolved in saline was administered perorally (p.o.) to two different groups (70 and $350 \mathrm{mg} / \mathrm{kg}$ ) once daily for 2 weeks after immunization. The normal control and CIA control mice were administered the same volume of saline.

Assessment of CIA. The clinical severity of CIA was quantified by three observers according to a graded scale from $0-4$, as displayed in Table I. Each paw and tail were scored and yielded a maximum possible score of 19 per animal.

Paw swelling was measured by average hind paw volume via water plethysmography (Model 7150; Ugo Basile Srl, Varese, Italy) on day 0 (D0) and day 35 after immunization. The increase in paw swelling was calculated by using the following formula: Paw swelling increase $(\%)=$ (paw swelling on D35 after immunization) - (paw swelling on D0 prior to immunization)/(paw swelling prior to immunization) x 100

Histopathological assessment of ankle joint. The ankle joints were removed on day 35 and fixed for $24 \mathrm{~h}$ in $4 \%$ paraformaldehyde. The fixed tissues were decalcified in Calci-Clear Rapid (National Diagnostics, Atlanta, GA, USA), embedded in paraffin and sectioned $(4 \mu \mathrm{m})$ using a microtome. Tissue sections were stained with hematoxylin and eosin and Safranin O/Fast Green staining (Sigma-Aldrich) to assess cartilage destruction. Bone destruction, vascular proliferation, synovial hyperplasia and inflammatory cell infiltration were assessed.

Spleen and thymus indexes. Mice were sacrificed by cervical dislocation after sampling for serum on day 35 after immunization. The wet spleen and thymus were weighed immediately following dissection. The indexes of the spleen and thymus 
Table II. Primers used in the current study.

\begin{tabular}{|c|c|}
\hline Gene & Sequences $\left(5^{\prime}-3^{\prime}\right)$ \\
\hline TNF- $\alpha$ & $\begin{array}{l}\text { GCGACGTGGAACTGGCAGAAG } \\
\text { TCCATGCCGTTGGTTAGGAGG }\end{array}$ \\
\hline IL-1 $\beta$ & $\begin{array}{l}\text { AAGCTCTCCACCTCAATGGACA } \\
\text { GTCTGCTCATTCACGAAAABBGAG }\end{array}$ \\
\hline IL-6 & $\begin{array}{l}\text { TCCAGTTGCCTTCTTGGGAC } \\
\text { GTGTAATTAAGCCTCCGACTTG }\end{array}$ \\
\hline IFN- $\gamma$ & $\begin{array}{l}\text { TCAAGTGGCATAGATGTCGAAGAA } \\
\text { TGGCTCTGCAGGATTTTCATG }\end{array}$ \\
\hline iNOS & $\begin{array}{l}\text { CTGCAGCACTTGGATCAGGAACCTG } \\
\text { GGGAGTAGCCTGTGTGCACCTGGAA }\end{array}$ \\
\hline $\mathrm{COX}-2$ & $\begin{array}{l}\text { TTGAAGACCAGGAGTACCGC } \\
\text { GGTACAGTCCCATGACATCG }\end{array}$ \\
\hline RANKL & $\begin{array}{l}\text { AAAACGCAGATTTGCAGGAC } \\
\text { GGCCACATCCAACCATGAG }\end{array}$ \\
\hline MMP-2 & $\begin{array}{l}\text { CCAGATCACATACAGGATCATTG } \\
\text { CTCCCAGCGTCCAAAGTT }\end{array}$ \\
\hline MMP-9 & $\begin{array}{l}\text { CTAAAGGCCATTCGAACACC } \\
\text { AAAGGCGTGTGCCAGAAG }\end{array}$ \\
\hline MMP-13 & $\begin{array}{l}\text { AGGTGACTGGCAAACTTGAT } \\
\text { CCAGAAGACCAGAAGGTCCA }\end{array}$ \\
\hline GAPDH & $\begin{array}{l}\text { CCACAGTCCATGCCATCAC } \\
\text { TCCACCACCCTGTTGCTGTA }\end{array}$ \\
\hline
\end{tabular}

TNF- $\alpha$, tumor necrosis factor; IL, interleukin; IFN- $\gamma$, interferon; iNOS, inducible nitric oxide synthase; COX-2, cyclooxygenase-2; RANKL, receptor activator of nuclear factor- $\kappa \mathrm{B}$ ligand; MMP, matrix metalloprotease; GAPDH, glyceraldehyde-3-phosphate dehydrogenase.

were calculated by using the following formula: Index = spleen and thymus weight of mouse / body weight of mouse.

Measurement of proinflammatory cytokines and $\operatorname{Ig} G 2 a$ levels. Levels of total tumor necrosis factor TNF- $\alpha$, interleukin (IL)-1 $\beta$, IL-6 and immunoglobulin $\mathrm{G}(\mathrm{IgG}) 2 \mathrm{a}$ in the serum of mice were investigated using commercially available enzyme-linked immunosorbent assay (ELISA) kits (cat nos. 560478, 559603, 555240 and 552576, respectively; BD Biosciences Pharmingen, San Diego, CA, USA). The aforementioned proinflammatory cytokines and IgG2a were measured according to the manufacturer's instructions, and concentrations were recalculated from their respective standard curves.

Measurement of prostaglandin E2 (PGE2) and nitric oxide (NO) levels. The level of PGE2 production in the serum of mice was measured using a commercially available ELISA kit (cat no. 514010; Cayman Chemical, Ann Arbor, MI, USA) according to the manufacturer's instructions.

Nitrite accumulation in the serum of mice was measured colorimetrically by the Griess reaction using a Griess reagent
(Sigma-Aldrich). For the assay, equal volumes of the serum of CIA mice and Griess reagent were mixed, and the absorption coefficient was calibrated using a sodium nitrite solution standard (Sigma-Aldrich). The absorbance of each sample after the Griess reaction was determined using a microplate reader at $540 \mathrm{~nm}$.

Reverse transcription-polymerase chain reaction (RT-PCR). Total RNA was extracted from joint tissues using TRIzol reagent (Invitrogen, Carlsbad, CA, USA) according to the manufacturer's instructions. The primers used to amplify TNF- $\alpha$, IL-1 $\beta$, IL-6, interferon (IFN)- $\gamma$, inducible NO synthase (iNOS), cyclooxygenase (COX)-2, RANKL, MMP-2, MMP-9, MMP-13 and glyceraldehyde-3-phosphate dehydrogenase (GAPDH) are shown in Table II.

For the experiment, $1 \mu \mathrm{g}$ of total RNA and $20 \mu \mathrm{l}$ of total reagents using One-step RT-PCR PreMix (iNtRON Biotechnology, Seongnam, Gyeonggi-do, Korea) was used. The PCR reaction was performed with a GeneAmp PCR System 9700 (Applied Biosystems; Thermo Fisher Scientific Inc., Waltham, MA, USA). The PCR products were separated by electrophoresis on $2 \%(\mathrm{w} / \mathrm{v})$ agarose gels and visualized with ethidium bromide. The quantity of mRNA was normalized to the amount of GAPDH, which was utilized as a housekeeping gene for each experimental condition. Bands were quantified by densitometry software (Scion Image version Beta 3b; Scion Corp., Frederick, MD, USA).

Statistical analysis. Statistical analysis was performed using GraphPad Prism 5 package (GraphPad Software Inc., San Diego, CA, USA). All data are expressed as mean \pm standard deviation. One-way analysis of variance followed by Dunnett's post-hoc multiple comparison tests was used to assess statistical significance. $\mathrm{P}<0.05$ was considered to indicate a statistically significant difference.

\section{Results}

Effect of FSE on macroscopic features of CIA. As displayed in Fig. 1A, significant increases in paw edema were recorded in CIA control mice following 2 intradermal injections of CII $(\mathrm{P}<0.001)$. In addition, macroscopic redness was observed, and a number of edemas were observed in several of the other joints of the CIA control mice. A significant reduction in paw edema was observed with FSE at 70 and $350 \mathrm{mg} / \mathrm{kg} /$ day, p.o. compared with the CIA control group $(\mathrm{P}<0.01$ and $\mathrm{P}<0.001$, respectively). The initial signs of arthritis development were visible between days 7 and 14 after primary immunization. The clinical scores of the CIA control group increased gradually after immunization, reaching a maximum score of $12.07 \pm 2.77$ by day 35 . Treatment with FSE at 70 and $350 \mathrm{mg} / \mathrm{kg} /$ day, p.o. resulted in significant attenuation of arthritis clinical scores $(\mathrm{P}<0.05$ and $\mathrm{P}<0.001$, respectively; Fig. 1B). The CIA control group revealed a considerable difference when compared with the normal group in paw swelling on day 35 . FSE reduced paw swelling in a dose-dependent manner when compared with the CIA control group (Fig. 1C).

Effect of FSE on histopathological changes. The current study conducted H\&E and Safranin O/Fast Green staining to 
A
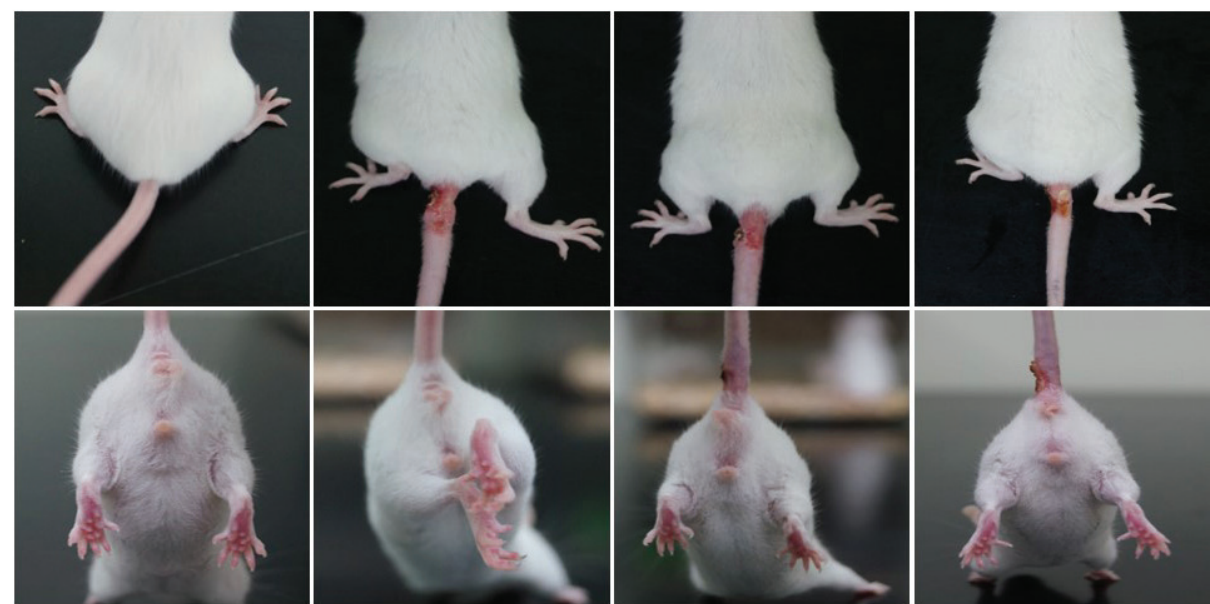

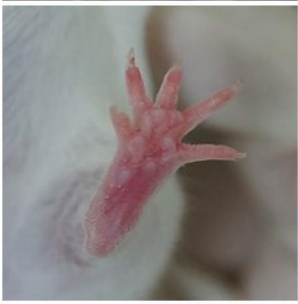

Normal control

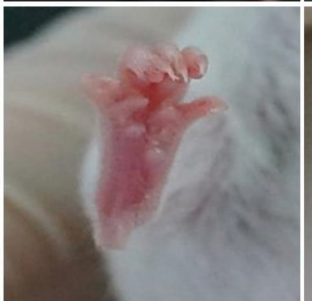

CIA control

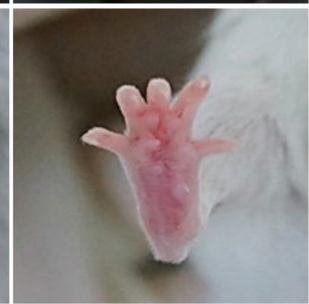

FSE $70 \mathrm{mg} / \mathrm{kg}$

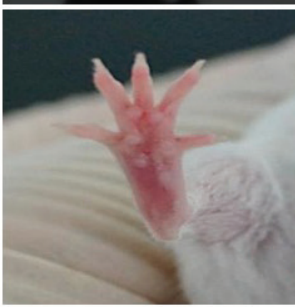

FSE $350 \mathrm{mg} / \mathrm{kg}$

$\mathbf{B}$

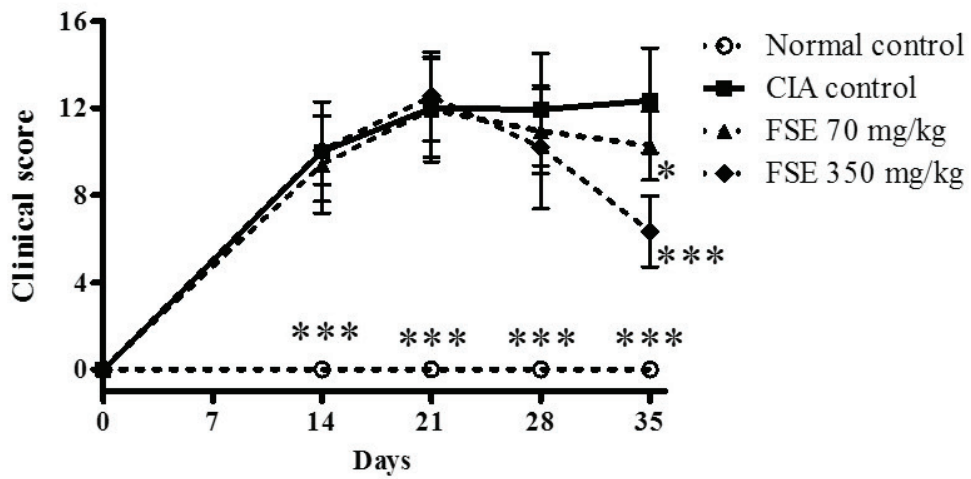

C
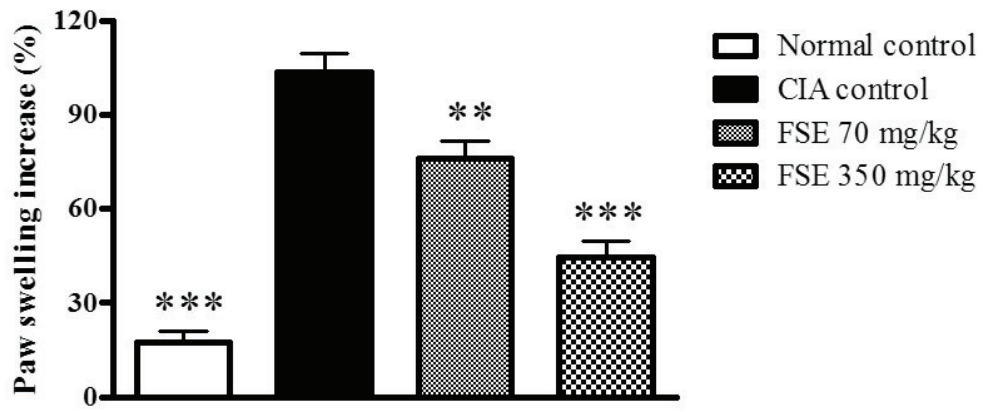

Figure 1. Effects of FSE on the development of CIA in BALB/c mice. (A) Representative clinical features on the tail and hind paws of mice on day 35. (B) The clinical severity of arthritis in each paw and tail was quantified according to a graded scale from 0 to 4 . Data are recorded as mean \pm S.D. from 5 animals. (C) Paw swelling was measured with a plethysmometer. Data are recorded as mean \pm standard deviation from 5 animals. ${ }^{*} \mathrm{P}<0.05,{ }^{* * *} \mathrm{P}<0.01,{ }^{* * * *} \mathrm{P}<0.001$ vs. CIA control group. CIA, type II collagen (CII)-induced arthritis; FSE, Fructus sophorae extract.

assess the effect of FSE on histological changes and cartilage destruction in the ankle joints. As indicated in Fig. 2, the normal control group displayed normal healthy articular space and tissues. In contrast, the CIA control group demonstrated synovial hyperplasia, destruction of articular cartilage and narrowed articular cavity. Groups treated with FSE $(70$ and $350 \mathrm{mg} / \mathrm{kg})$ displayed a marked reduction in synovial hyperplasia, destruction of articular cartilage and articular cavity changes (such as increased protrusion of synovial villi into the articular cavity) when compared with the CIA control group. 


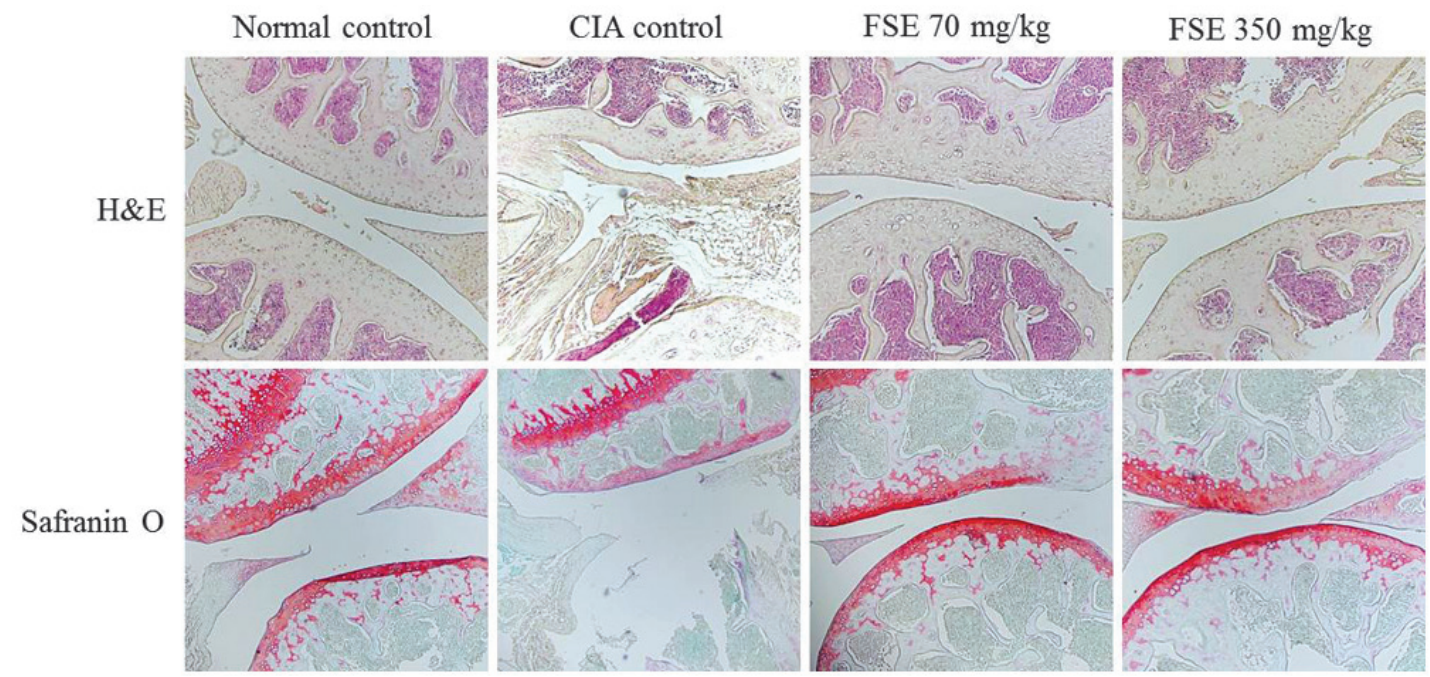

Figure 2. Histological observations of ankle joints. Representative histological sections of ankle joints stained with H\&E (magnification, $\mathrm{x} 40$ ) and Safranin O/Fast Green (magnification, x40). The normal control group displayed normal, healthy articular space and tissues. The CIA control group reported synovial hyperplasia, destruction of articular cartilage and close articular cavity. FSE-treated mice groups (70 and $350 \mathrm{mg} / \mathrm{kg}$ ) revealed reduction in pathological events including synovial hyperplasia, destruction of articular cartilage and articular cavity changes compared with the CIA control group. H\&E, hematoxylin and eosin; CIA, type II collagen (CII)-induced arthritis; FSE, Fructus sophorae extract.

Effect of FSE on spleen and thymus indexes and level of serum $\operatorname{IgG2a}$. To examine the effects of FSE on the immune response in CIA mice, the current study investigated spleen and thymus indexes and levels of serum IgG2a. The CIA control group differed from the normal control group, displaying significantly high spleen and thymus indexes. FSE reduced the indexes of spleen and thymus when compared with those of the CIA control group $(\mathrm{P}<0.01$ and $\mathrm{P}<0.05$, respectively). However, FSE (350 mg/kg/day p.o.) did not induce a significantly lower thymus index compared with the CIA control group (Fig. 3A). The serum IgG2a level, which is known to be pathogenic in CIA (15) was also evaluated. The CIA control group had increased serum $\mathrm{IgG} 2 \mathrm{a}$ levels relative to those of the normal control group $(\mathrm{P}<0.05)$, whereas the FSE ( $350 \mathrm{mg} / \mathrm{kg} /$ day p.o.) group displayed suppressed serum IgG2a levels compared with those of the CIA control group ( $\mathrm{P}<0.05$; Fig. 3B).

FSE suppresses production and mRNA expression levels of proinflammatory cytokines. RA is a systemic autoimmune disease, therefore circulating proinflammatory cytokine levels were measured in the serum and mRNA expression levels of these genes were investigated in joint tissues obtained on day 35. The CIA control group revealed increases in the levels of TNF- $\alpha$, IL- $1 \beta$, IL- 6 and IFN- $\gamma$ when compared with those of the normal control group, whereas FSE suppressed these cytokines when compared with those of the CIA control group $(\mathrm{P}<0.05, \mathrm{P}<0.01$ and $\mathrm{P}<0.001$, respectively; Fig. 4A). Consistent with results in the serum, significant induction of the TNF- $\alpha$, IL-1 $\beta$, IL- 6 and IFN- $\gamma$ genes was observed in joint tissues of the CIA control group in comparison with the normal control group $(\mathrm{P}<0.001)$. FSE downregulated the activation of these genes in a dose-dependant manner compared with the CIA control group $(\mathrm{P}<0.05$ and $\mathrm{P}<0.001$, respectively; Fig. 4B).

Effect of FSE on PGE2 and NO production and mRNA expression levels of COX-2 and iNOS. PGE2 and NO, produced by
COX-2 and iNOS, respectively, are important inflammatory mediators involved in the pathogenesis of RA. Levels of PGE2 and NO, the metabolites of COX-2 and iNOS, were evaluated in the serum and mRNA expression levels of COX-2 and iNOS were investigated in joint tissue obtained on day 35 . A significant increase in PGE2 and NO production was observed in the CIA control group. FSE significantly reduced the levels of PGE2 and NO in a dose-dependent manner when compared with those of the CIA control group (Fig. 5A and B). Consistent with results in the serum, a marked induction of the COX-2 and iNOS genes was observed in the joint tissues of the CIA control group in comparison with the normal control group. FSE downregulated the expression of COX-2 (both treatments) and iNOS ( $350 \mathrm{mg} / \mathrm{kg}$ group) when compared with those of the CIA control group (Fig. 5C).

Effect of FSE on the mRNA expression levels of RANKL and MMPs. The mRNA expression of RANKL, MMP-2, MMP-9 and MMP-13 genes was investigated in joint tissue obtained on day 35. Significant induction of the RANKL, MMP-2, MMP-9 and MMP-13 genes was observed in the joint tissues of the CIA control group compared with those of the normal control group $(\mathrm{P}<0.001)$. Treatment with 70 or $350 \mathrm{mg} / \mathrm{kg}$ FSE significantly downregulated expression of RANKL $(\mathrm{P}<0.001)$ MMP-2, MMP-9 and MMP-13 ( $\mathrm{P}<0.05$ or $\mathrm{P}<0.001)$ compared with the CIA control group (Fig. 6). These findings demonstrate that FSE suppresses synovial inflammation, cartilage damage and bone destruction.

\section{Discussion}

Fructus sophorae, the dried ripe fruit of S.japonicum (L.), is an herb for clearing heat (reducing fever), purging Pathogenic Fire and cooling the blood to stop bleeding in Korean and Chinese medicine, and has traditionally been used for its hemostatic properties (1). The current study revealed that some components in Fructus sophorae have hemostatic properties as well as 
A
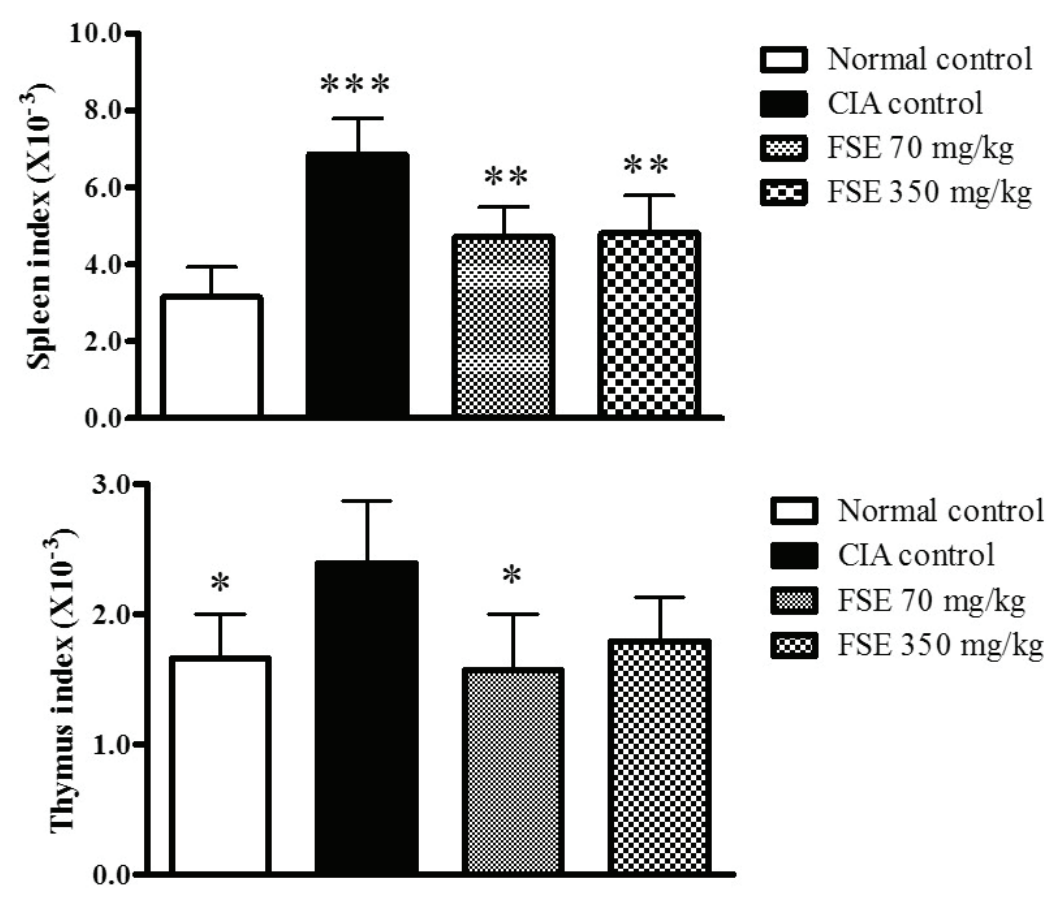

B
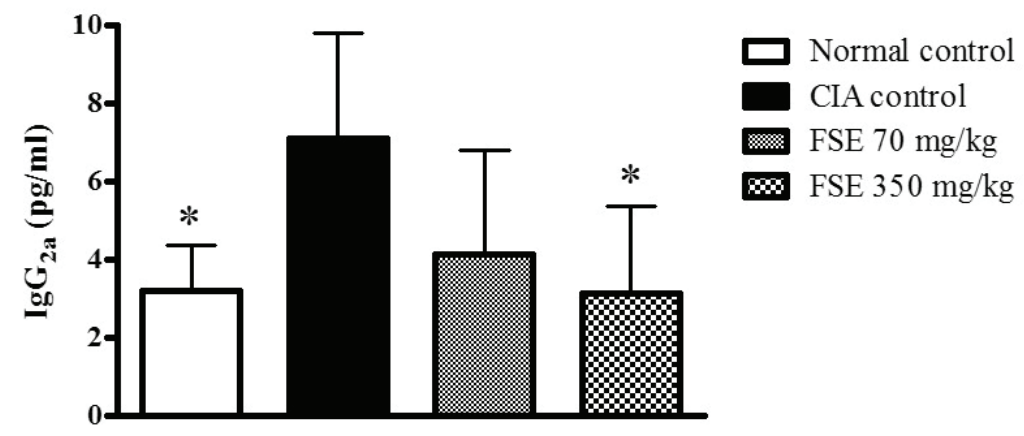

Figure 3. Effects of FSE on spleen and thymus indexes and serum IgG2a levels in CIA-positive BALB/c mice. (A) Index $=$ Spleen or thymus weight of mouse/body weight of mouse. (B) Serum IgG2a level. Data are recorded as mean \pm standard deviation from 5 animals. ${ }^{*} \mathrm{P}<0.05,{ }^{* * *} \mathrm{P}<0.01,{ }^{* * * *} \mathrm{P}<0.001$ vs. CIA control group. CIA, type II collagen (CII)-induced arthritis; FSE, Fructus sophorae extract.

anti-obesity, anti-tumor, anti-menopausal and anti-hemorrhoid effects (16-18). However, a limited amount of research has investigated whether Fructus sophorae has therapeutic effects on RA.

In the present study, it was determined that FSE had potentially protective effects in a CII-induced CIA mouse model. The CIA mouse model is a useful animal model for the assessment of RA characterized by synovitis, leading to the progressive destruction of cartilage and bone (14).

The initial clinical indicators of CIA were observed to be articular weakness, periarticular erythema and edema in the hind paws. FSE reduced the clinical symptoms, including arthritis clinical scores and paw swelling of CIA mice, in a dose-dependent manner (Fig. 1). Histological observations supported the protective effect of FSE against the progress of the pathology of CIA, including synovial hyperplasia, a narrowing joint space and cartilage erosion (Fig. 2). The aforementioned results suggest that FSE has an inhibitory effect against the clinical pathological development of CIA.

As RA is an autoimmune disorder, the effect of FSE on immune organs was also assessed and it was established that
FSE downregulated the spleen and thymus indexes in mice with CIA (Fig. 3A).

With regard to the pathological changes associated with CIA, the immune and inflammatory systems demonstrated increased serum levels of $\operatorname{IgG} 2 \mathrm{a}$, proinflammatory cytokines, T helper (Th) 1-mediated autoimmune component IFN- $\gamma$ and other inflammatory mediators. In addition, this system induces an increase in gene expression associated with synovial inflammation, cartilage and bone destruction.

The B cell, which is predominantly mediated by the $\mathrm{IgG} 2$ isotype of anti-CII immunoglobulin, is critical to the development of CIA $(15,19)$. In the current study, FSE inhibited serum IgG2a levels in a dose-dependent manner (Fig. 3B). This suggests that FSE may reduce joint damage by inhibiting the production of $\mathrm{IgG} 2 \mathrm{a}$ by controlling the organs of the immune system, including the spleen and the thymus in CIA pathology.

Proinflammatory cy tokines, in particular TNF- $\alpha$, IL-1 and IL-6, are expressed in joints and serve an important role in the development of collagen-induced arthritis $(6,7)$. The aforementioned cytokines contribute to synovial inflammation by inducing the production of other cytokines, chemokines 
A
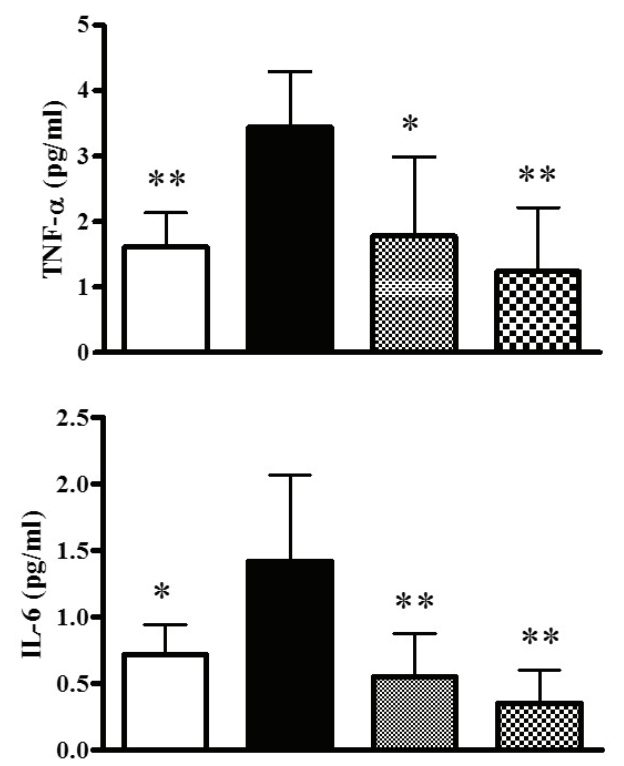

B

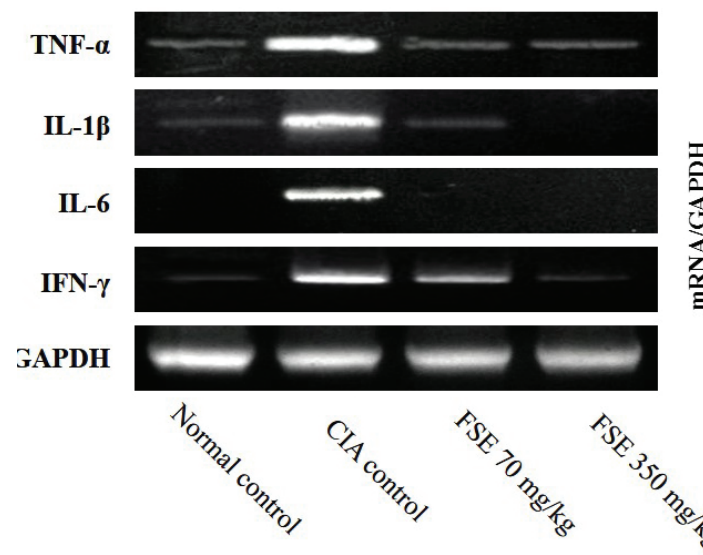

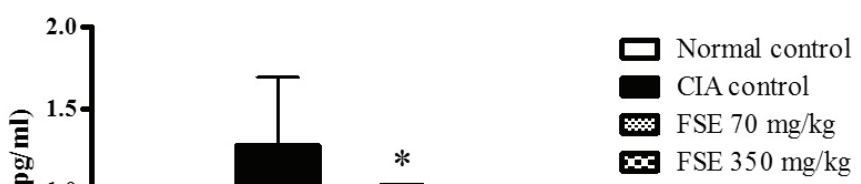
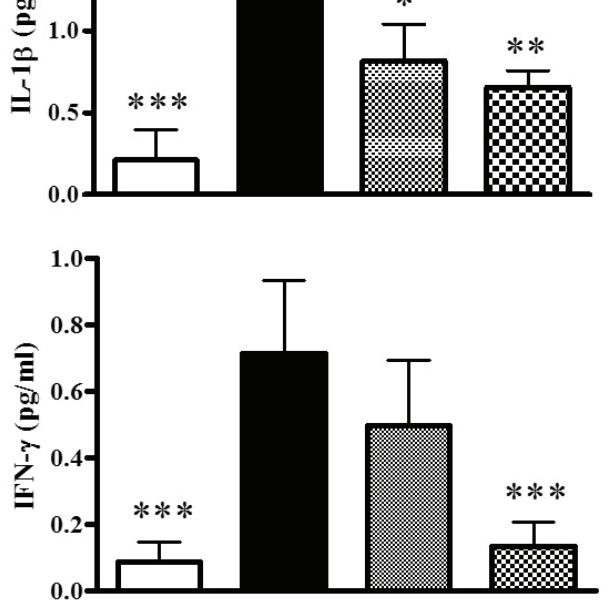

$\square$ Normal control

CIA control

FSE $70 \mathrm{mg} / \mathrm{kg}$

$\$$ FSE $350 \mathrm{mg} / \mathrm{kg}$

Figure 4. Effects of FSE on serum levels of IL-1 $\beta$, IL-6, TNF- $\alpha$ and INF- $\gamma$ and mRNA expression levels of IL-1 $\beta$, IL-6, TNF- $\alpha$ and INF- $\gamma$ from joint tissue in a CIA mouse model. (A) Serum IL-1 $\beta$, IL-6, TNF- $\alpha$ and INF- $\gamma$ levels. Data are recorded as mean \pm standard deviation from 5 animals. (B) Representative IL-1 $\beta$, IL-6, TNF- $\alpha$ and INF- $\gamma$ mRNA expression level, quantified by the densitometric analysis of bands and normalized to those of GAPDH mRNA. Data are recorded as mean \pm standard deviation from 3 experiments on joint tissues. ${ }^{*} \mathrm{P}<0.05,{ }^{* *} \mathrm{P}<0.01,{ }^{* * * *} \mathrm{P}<0.001$ vs. CIA control group. CIA, type II collagen (CII)-induced arthritis; FSE, Fructus sophorae extract; IL, interleukin; TNF, tumor necrosis factor; IFN, interferon; GAPDH, glyceraldehyde 3-phosphate dehydrogenase.

and small proinflammatory mediators (for example PGE2 and NO) (20). It has been determined that these cytokines also participate in the destruction of bone and cartilage by inducing expression of RANKL and MMPs in RA $(6,21,22)$. IFN- $\gamma$ produced by CD4 Th1 cells is a potent inducer of the inflammatory response in CIA (15). In the present study, FSE inhibited serum levels and gene expression of proinflammatory cytokines TNF- $\alpha$, IL-1 and IL- 6 and Th1-mediated cytokine IFN- $\gamma$ (Fig. 4). This suggests that FSE contributes to anti-inflammatory action by inhibiting serum proinflammatory cytokine release and the mRNA expression levels of proinflammatory cytokines in ankle joint tissue.

PGE2 and NO are synthesized in excess from the synovium of inflamed joints and exacerbate joint damage in RA. PGE2 and COX-2 induce vasodilation, fluid extravasation and pain in synovial tissue, and are implicated in the development of erosions of articular cartilage and bone (8). NO causes $\mathrm{T}$ cell dysfunction and contributes to bone loss in patients with RA $(10,23,24)$. Evidence from previous arthritis studies reported that the inhibition of NO and iNOS mRNA levels was effective in the treatment of RA (25). In the current study, FSE significantly inhibited serum levels of PGE2 and NO and gene expression of COX-2 and iNOS in a dose-dependent manner (Fig. 5). This suggests that FSE contributed to anti-inflammatory action via inhibition of serum PGE2 and NO release, in addition to mRNA expression levels of COX-2 and iNOS in ankle joint tissue.

RANKL and MMPs are potent biomarkers associated with bone and cartilage degradation. RANKL is critical for osteoclastogenesis, and regulates the differentiation, maturation and bone resorptive activity of osteoclasts (12). Expression of RANKL is regulated by pro-inflammatory cytokines TNF- $\alpha$, IL- $1 \beta$ and IL-6, as well as inflammatory mediators such as PGE2 (26). MMPs are a family of zinc-dependent and calcium-dependent proteolytic enzymes secreted by the resident cells and invading cells of joint tissues (27). MMPs are also induced by cytokines 
A
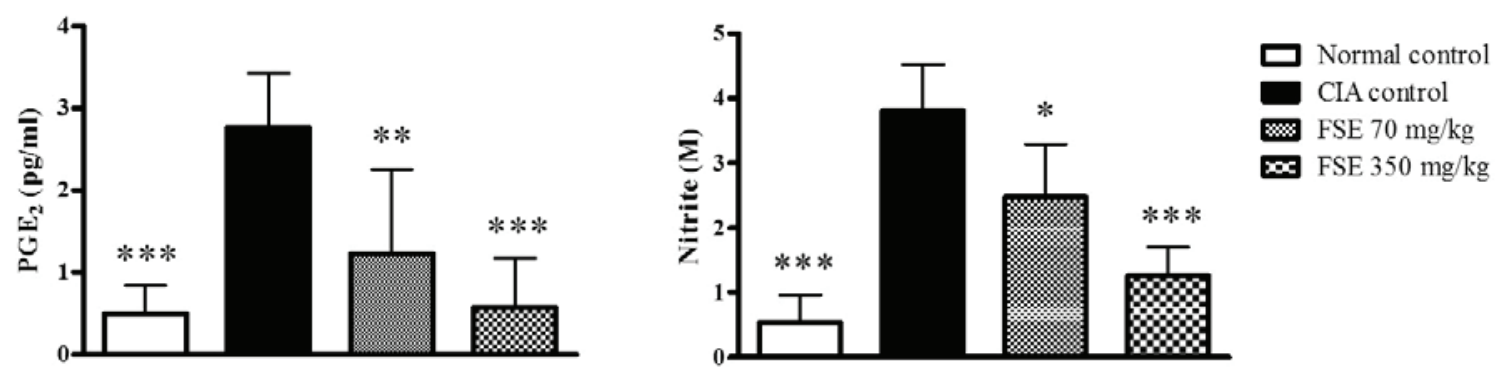

B

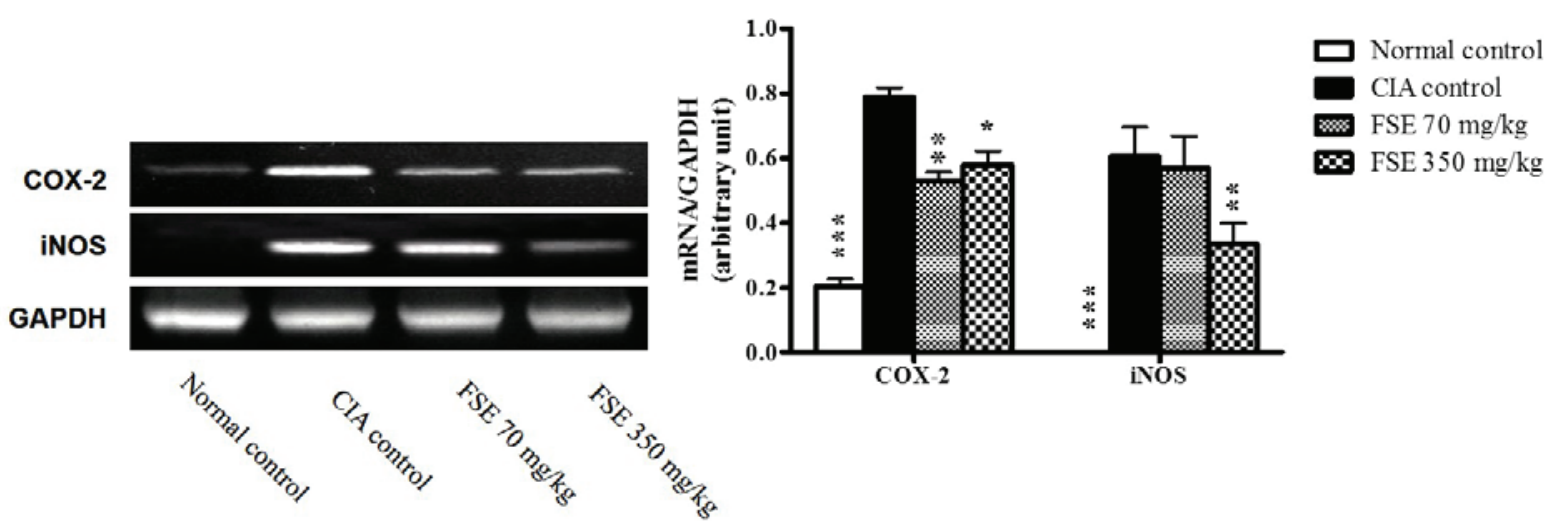

Figure 5. Effects of FSE on serum levels of PGE2 and NO, and mRNA expression levels of COX-2 and iNOS from CIA joint tissue in BALB/c mice. (A) Serum PGE2 and NO levels. Data are recorded as mean \pm standard deviation from 5 animals. (B) Representative COX-2 and iNOS mRNA expression level. Data are recorded as mean \pm standard deviation from 3 experiments. ${ }^{*} \mathrm{P}<0.05,{ }^{* * *} \mathrm{P}<0.01,{ }^{* * * *} \mathrm{P}<0.001$ vs. CIA control group. CIA, type II collagen (CII)-induced arthritis; FSE, Fructus sophorae extract; PGE2, prostaglandin E2; COX-2, cyclooxygenase-2; iNOS, inducible nitric oxide synthase; GAPDH, glyceraldehyde 3-phosphate dehydrogenase.

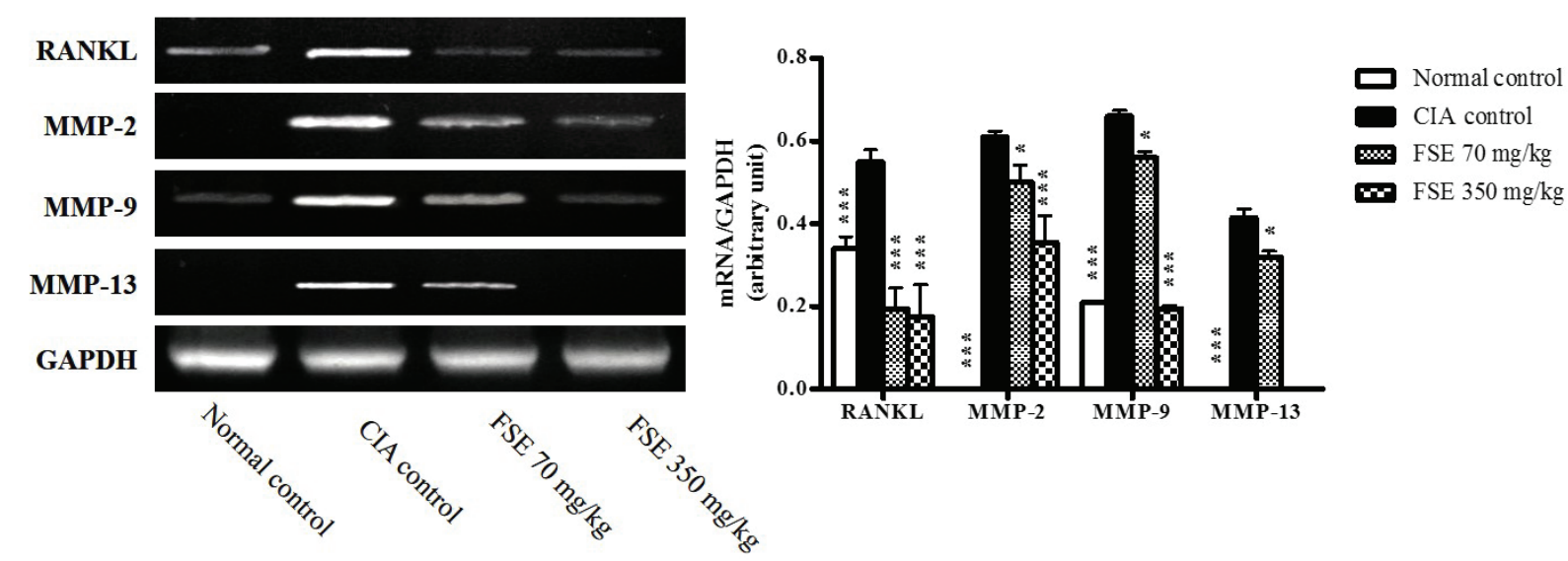

Figure 6. Effects of FSE on mRNA expression levels of RANKL, MMP-2, MMP-9, and MMP-13 from joint tissue in CIA mice model. Representative RANKL, MMP-2, MMP-9, and MMP-13 mRNA expression level. The quantity of autoradiograph bands was conducted as described in Fig. 4. Data are recorded as mean \pm standard deviation from 3 experiments on joint tissues. Statistics were determined as described in Fig. $1 .{ }^{*} \mathrm{P}<0.05,{ }^{* * *} \mathrm{P}<0.001 \mathrm{vs}$. CIA control group. CIA, type II collagen (CII)-induced arthritis; FSE, Fructus sophorae extract; RANKL, receptor activator of nuclear factor- $\mathrm{B}$ ligand; MMP, matrix metalloprotease; GAPDH, glyceraldehyde 3-phosphate dehydrogenase.

and growth factors and serve crucial roles in remodeling connective tissue and degrading the extracellular matrix (28-30). The gelatinases MMP-2 and MMP-9 and the collagenase MMP-13 are important in arthritic disease and cleave native fibrillar collagen and degrade the extracellular matrix molecules of articular cartilage in RA $(31,32)$. In the current study, FSE inhibited gene expression of RANKL, MMP-2, MMP-9 and MMP-13 in the inflamed RA joint tissue (Fig. 6). This suggests that FSE protects the cartilage and bone damage by inhibiting the gene activation of RANKL and MMPs in RA joint tissue.

The present study has demonstrated that FSE has a protective effect against disease progression in a mouse model of CIA. This effect is associated with the suppression of factors associated with the rheumatoid inflammatory response by FSE, synovial inflammation and cartilage and bone damage. The present results suggest that FSE may provide potential 
protection against inflammation and bone and cartilage damage in RA and, as such, would be a valuable candidate for further investigation as a novel anti-arthritic agent.

In conclusion, the aim of the current study was to determine whether FSE has anti-arthritic effects. The anti-arthritic protective effects of FSE were identified, and the effects were observed to be associated with the suppression of inflammatory actions and protection of cartilage and bone damage in the mouse model of CIA.

\section{Acknowledgements}

The present study was supported by the R\&D program of MOTIE/KIAT (Establishment of Infra Structure for Anti-aging Industry Support; Seoul, Korea; grant no. N0000697)

\section{References}

1. Gan T, Liu YD, Wang Y and Yang J: Traditional Chinese Medicine herbs for stopping bleeding from haemorrhoids. Cochrane Database Syst Rev 10: CD006791, 2010.

2. Joo SS, Won TJ, Kang HC and Lee DI: Isoflavones extracted from Sophorae fructus upregulate IGF-I and TGF-beta and inhibit osteoclastogenesis in rat bone marrow cells. Arch Pharm Res 27: 99-105, 2004.

3. Shim JG, Yeom SH, Kim HJ, Choi YW,Lee DI, Song KY, Kwon SH and Lee MW: Bone loss preventing effect of Sophorae Fructus on ovariectomized rats. Arch Pharm Res 28: 106-110, 2005.

4. Joo SS, Kang HC, Lee MW, Choi YW and Lee DI: Inhibition of IL-1beta and IL-6 in osteoblast-like cell by isoflavones extracted from Sophorae fructus. Arch Pharm Res 26: 1029-1035, 2003.

5. Scott DL, Wolfe F and Huizinga TW: Rheumatoid arthritis. Lancet 376: 1094-1108, 2010.

6. McInnes IB and Schett G: Cytokines in the pathogenesis of rheumatoid arthritis. Nat Rev Immunol 7: 429-442, 2007.

7. Brennan FM and McInnes IB: Evidence that cytokines play a role in rheumatoid arthritis. J Clin Invest 118: 3537-3545, 2008.

8. Fattahi MJ and Mirshafiey A: Prostaglandins and rheumatoid arthritis. Arthritis 2012: 239310, 2012.

9. Cillero-Pastor B, Martin MA, Arenas J, López-Armada MJ and Blanco FJ: Effect of nitric oxide on mitochondrial activity of human synovial cells. BMC Musculoskelet Disord 12: 42, 2011.

10. Nagy G, Koncz A, Telarico T, Fernandez D, Ersek B, Buzás E and Perl A: Central role of nitric oxide in the pathogenesis of rheumatoid arthritis and systemic lupus erythematosus. Arthritis Res Ther 12: 210, 2010.

11. Karmakar S, Kay J and Gravallese EM: Bone damage in rheumatoid arthritis: Mechanistic insights and approaches to prevention. Rheum Dis Clin North Am 36: 385-404, 2010.

12. Schett G, Hayer S, Zwerina J, Redlich K and Smolen JS: Mechanisms of Disease: The link between RANKL and arthritic bone disease. Nat Clin Pract Rheumatol 1: 47-54, 2005.

13. Udagawa N, Kotake S, Kamatani N, Takahashi N and Suda T: The molecular mechanism of osteoclastogenesis in rheumatoid arthritis. Arthritis Res 4: 281-289, 2002.

14. Brand DD, Latham KA and Rosloniec EF: Collagen-induced arthritis. Nat Protoc 2: 1269-1275, 2007.

15. Brand DD, Kang AH and Rosloniec EF: Immunopathogenesis of collagen arthritis. Springer Semin Immunopathol 25: 3-18, 2003.
16. Chang L, Ren Y, Cao L, Sun Y, Sun Q, Sheng N, Yuan L, Zhi X and Zhang L: Simultaneous determination and pharmacokinetic study of six flavonoids from Fructus Sophorae extract in rat plasma by LC-MS/MS. J Chromatogr B Analyt Technol Biomed Life Sci 904: 59-64, 2012.

17. Ha do T, Trung TN, Phuong TT, Yim N, Chen QC and Bae K: The selected flavonol glycoside derived from Sophorae Flos improves glucose uptake and inhibits adipocyte differentiation via activation AMPK in 3T3-L1 cells. Bioorg Med Chem Lett 20: 6076-6081, 2010.

18. Park KW, Lee JE and Park KM: Diets containing Sophora japonica L. prevent weight gain in high-fat diet-induced obese mice. Nutr Res 29: 819-824, 2009.

19. Brand DD, Marion TN, Myers LK, Rosloniec EF, Watson WC, Stuart JMand Kang AH: Autoantibodies to murine type II collagen in collagen-induced arthritis: A comparison of susceptible and nonsusceptible strains. J Immunol 157: 5178-5184, 1996.

20. Feldmann M and Maini RN: Anti-TNF alpha therapy of rheumatoid arthritis: What have we learned? Annu Rev Immunol 19: 163-196, 2001.

21. Dayer JM: The pivotal role of interleukin-1 in the clinical manifestations of rheumatoid arthritis. Rheumatology (Oxford) 42 (Suppl 2): ii3-ii10, 2003.

22. Hashizume M and Mihara M: The roles of interleukin-6 in the pathogenesis of rheumatoid arthritis. Arthritis 2011: 765624, 2011.

23. Nagy G, Clark JM, Buzás EI, Gorman CL and Cope AP: Nitric oxide, chronic inflammation and autoimmunity. Immunol Lett 111: 1-5, 2007.

24. Mäki-Petäjä KM, Cheriyan J, Booth $\mathrm{AD}$, Hall FC, Brown J, Wallace SM, Ashby MJ, McEniery CM and Wilkinson IB: Inducible nitric oxide synthase activity is increased in patients with rheumatoid arthritis and contributes to endothelial dysfunction. Int J Cardiol 129: 399-405, 2008.

25. Wang B, Ma L, Tao X and Lipsky PE: Triptolide, an active component of the Chinese herbal remedy Tripterygium wilfordii Hook F, inhibits production of nitric oxide by decreasing inducible nitric oxide synthase gene transcription. Arthritis Rheum 50: 2995-3303, 2004.

26. Kotake S, Udagawa N, Takahashi N, Matsuzaki K, Itoh K, Ishiyama S, Saito S, Inoue K, Kamatani N, Gillespie MT, et al: IL-17 in synovial fluids from patients with rheumatoid arthritis is a potent stimulator of osteoclastogenesis. J Clin Invest 103: $1345-1352,1999$

27. Murphy G, Knäuper V, Atkinson S, Butler G, English W, Hutton M, Stracke J and Clark I: Matrix metalloproteinases in arthritic disease. Arthritis Res 4 (Suppl 3): S39-S49, 2002.

28. Nagase $\mathrm{H}$ and Woessner JF Jr: Matrix metalloproteinases. J Biol Chem 274: 21491-21494, 1999.

29. Vu TH and Werb Z: Matrix metalloproteinases: Effectors of development and normal physiology. Genes Dev 14: 2123-2133, 2000.

30. Chang YH, Lin IL, Tsay GJ, Yang SC, Yang TP, Ho KT, Hsu TC and Shiau MY: Elevated circulatory MMP-2 and MMP-9 levels and activities in patients with rheumatoid arthritis and systemic lupus erythematosus. Clin Biochem 41: 955-959, 2008.

31. Birkedal-Hansen H: Proteolytic remodeling of extracellular matrix. Curr Opin Cell Biol 7: 728-735, 1995.

32. Kim KS, Choi HM, Lee YA, Choi IA, Lee SH, Hong SJ, Yang HI and Yoo MC: Expression levels and association of gelatinases MMP-2 and MMP-9 and collagenases MMP-1 and MMP-13 with VEGF in synovial fluid of patients with arthritis. Rheumatol Int 31: 543-547, 2011. 